\title{
Evolutionary history of the vertebrate Piwi gene family
}

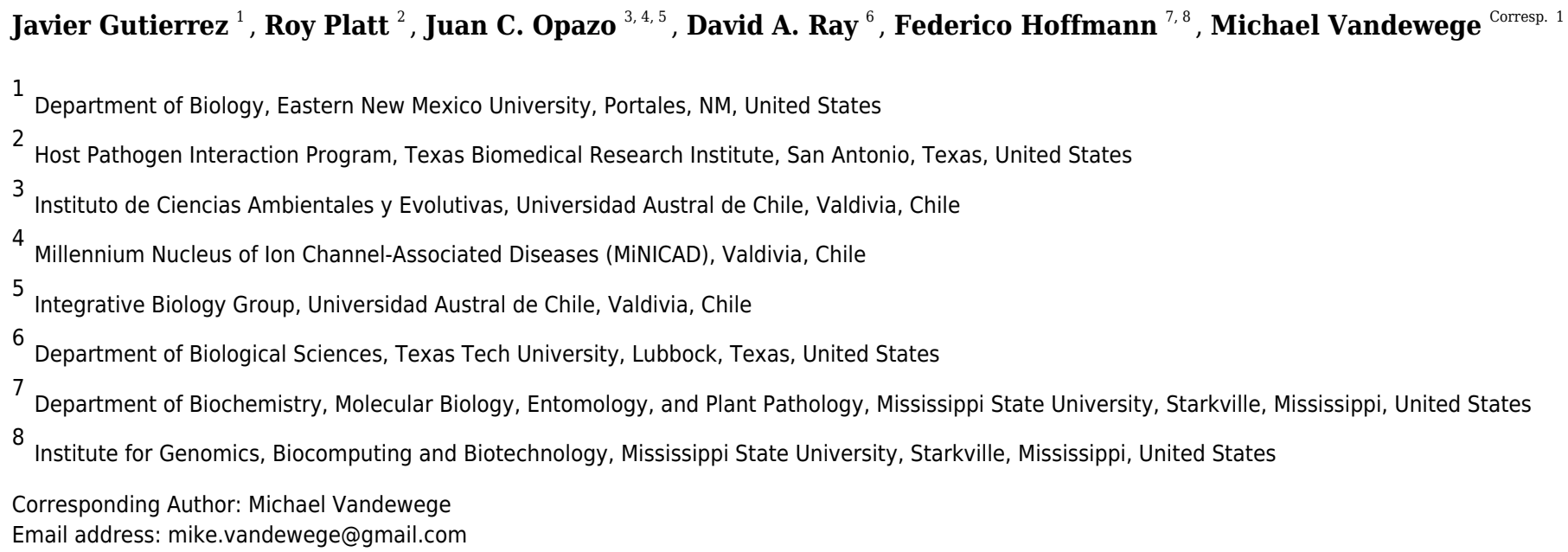

PIWIs are regulatory proteins that belong to the Argonaute family. Piwis are primarily expressed in gonads and protect the germline against the mobilization and propagation of transposable elements (TEs) through transcriptional gene silencing. Vertebrate genomes encode up to four Piwi genes: Piwil1, Piwil2, Piwil3 and Piwil4, but their duplication history is unresolved. We leveraged phylogenetics, synteny and expression analyses to address this void. Our phylogenetic analysis suggests Piwil1 and Piwil2 were retained in all vertebrate members. Piwil4 was the result of Piwil1 duplication in the ancestor of gnathostomes, but was independently lost in ray-finned fishes and birds. Further, Piwil3 was derived from a tandem Piwill duplication in the common ancestor of marsupial and placental mammals, but was secondarily lost in Atlantogenata (Xenarthra and Afrotheria) and some rodents. The evolutionary rate of Piwil3 is considerably faster than any Piwi among all lineages, but an explanation is lacking. Our expression analyses suggest Piwi expression has mostly been constrained to gonads throughout vertebrate evolution. Vertebrate evolution is marked by two early rounds of whole genome duplication and many multigene families are linked to these events. However, our analyses suggest Piwi expansion was independent of whole genome duplications. 
1 Evolutionary history of the vertebrate Piwi gene family

2 Javier Gutierrez ${ }^{1}$, Roy N. Platt II², Juan C. Opazo ${ }^{3,4,5}$, David A. Ray ${ }^{6}$, Federico G. Hoffmann ${ }^{7,8}$,

3 Michael W. Vandewege ${ }^{1}$

$4{ }^{1}$ Department of Biology, Eastern New Mexico University, Portales, New Mexico, USA.

5

$6{ }^{2}$ Host Pathogen Interaction Program, Texas Biomedical Research Institute, San Antonio TX,

7 USA

8

${ }^{3}$ Instituto de Ciencias Ambientales y Evolutivas, Facultad de Ciencias, Universidad Austral de Chile, Valdivia, Chile.

${ }^{4}$ Millennium Nucleus of Ion Channel-Associated Diseases (MiNICAD), Valdivia, Chile.

Integrative Biology Group, Universidad Austral de Chile, Valdivia, Chile.

${ }^{6}$ Department of Biological Sciences, Texas Tech University, Lubbock, Texas, USA

7Department of Biochemistry, Molecular Biology, Entomology, and Plant

Pathology, Mississippi State University, Starkville, Mississippi, USA.

8Institute for Genomics, Biocomputing and Biotechnology, Mississippi State

University, Starkville, Mississippi, USA.

\section{Corresponding author:}

Michael W. Vandewege

Department of Biology

Eastern New Mexico University

1500 S Avenue K, Portales, NM 88130, USA

Email address: mike.vandewege@gmail.com

PIWIs are regulatory proteins that belong to the Argonaute family. Piwis are primarily expressed in gonads and protect the germline against the mobilization and propagation of transposable elements (TEs) through transcriptional gene silencing. Vertebrate genomes encode up to four Piwi genes: Piwil1, Piwil2, Piwil3 and Piwil4, but their duplication history is unresolved. We leveraged phylogenetics, synteny and expression analyses to address this void. Our phylogenetic analysis suggests Piwil1 and Piwil2 were retained in all vertebrate members. Piwil4 was the result of Piwil1 duplication in the ancestor of gnathostomes, but was independently lost in ray-finned fishes and birds. Further, Piwil3 was derived from a tandem 
42 Piwil1 duplication in the common ancestor of marsupial and placental mammals, but was

43 secondarily lost in Atlantogenata (Xenarthra and Afrotheria) and some rodents. The evolutionary

44 rate of Piwil3 is considerably faster than any Piwi among all lineages, but an explanation is

45 lacking. Our expression analyses suggest Piwi expression has mostly been constrained to

46 gonads throughout vertebrate evolution. Vertebrate evolution is marked by two early rounds of

47 whole genome duplication and many multigene families are linked to these events. However,

48 our analyses suggest Piwi expansion was independent of whole genome duplications.

49

50

51

52

53

54

55

56

57

58

59

60

61

62

63

64

65

66

67

68

69

70

71

72

73

74

75

76

77

78

79

80

81

82

83

\section{Introduction}

The Piwi gene family has become one of the more charismatic gene families in recent decades due to their role in defending the genome against transposable elements (TEs) and viruses (Siomi et al. 2011; Sun et al. 2017; Kolliopoulou et al. 2019). PIWI proteins belong to a larger family of Argonaute proteins that include two major clades, the AGOs and PIWIs (Carmell et al. 2002). All members of the Argonaute family contain a PAZ and PIWI domain and participate in RNA-induced silencing complexes (RISCs) with small RNAs. AGOs are ubiquitously expressed and bind microRNAs (Tang 2005) to target and silence mRNAs. In vertebrates, PIWI proteins are generally restricted to the germline, bind their own class of small RNAs, PIWI interacting RNAs (piRNAs), and regulate TEs and viruses through transcriptional silencing (Girard et al. 2006; Lau et al. 2006; Brennecke et al. 2007; Sun et al. 2017). The details of PIWI function vary among animals, but most seem to be involved in a model described as the 'ping-pong cycle' where PIWIs cleave RNA into piRNAs, the cleaved piRNAs form riboprotein complexes with the PIWIs and direct these complexes to bind complementary transcripts. These complexes can then cleave newly bound transcripts and repeat the cycle. Secondary piRNAs can also be used to guide PIWI proteins to TE loci in the genome and initiate methylation (Aravin et al. 2008; Kuramochi-Miyagawa et al. 2008; Rozhkov et al. 2013). In addition to the ping-pong cycle, the diversity of piRNAs is increased by 5'-to-3' phasing from the site of initial piRNA formation (Han et al. 2015; Mohn et al. 2015; Ozata et al. 2019).

The bulk of vertebrate PIWI protein functional analyses are restricted to experiments in mice. From these experiments, we understand that the expression of Piwis varies temporally during germ cell development and spermatogenesis. Piwil2 (Mili) is the first Piwi to become expressed at embryonic day 12.5 (E12.5) in developing testes and is linked to the post-transcriptional silencing of TEs (Aravin et al. 2007). Piwil4 (Miwi2) is expressed between E14.5 and post-natal day 3 (P3). piRNAs from PIWIL2 are loaded onto PIWIL4 and PIWIL4 initiates the de novo establishment of methylation marks among TE loci in gonocytes during this time (Carmell et al. 2007; Aravin et al. 2008; Molaro et al. 2014; Zoch et al. 2020). PIWIL4 is also expressed in undifferentiated spermatogonia in adults, although the link to TEs in these cell types is still under investigation (Carrieri et al. 2017; Vasiliauskaite et al. 2018). Piwil1 (Miwi) is the last Piwi gene expressed at approximately P14, during the pachytene stage of prophase I (Girard et al. 2006). However, most piRNAs associated with PIWIL1 are derived from non-coding regions and PIWIL1 plays a relevant role cleaving/removing mRNAs during later stages of spermatogenesis (Reuter et al. 2011; Li et al. 2013; Gou et al. 2015; Wu et al. 2020). Some placental mammals, including Primates and Laurasiatherians have a 4th paralog, Piwil3. Piwil3 is the least studied of the four paralogs, likely because this paralog is absent from the mouse genome. However,

Peer] reviewing PDF | (2021:06:62032:1:0:NEW 25 Sep 2021) 
84 experiments in cattle suggest Piwil3 is largely expressed in oocytes and early embryos and

85

86

87

88

89

90

91

92

93

94

95

96

97

98

99

100

101

102

103

104

105

106

107

108

109

110

111

112

113

114

115

116

117

118

119

120

121

PIWIL3 generates TE derived ping-pong piRNAs (Roovers et al. 2015; Tan et al. 2020).

The copy number of the Piwi gene family varies among animals, for example C.elegans encodes two Piwis from a lineage specific duplication (Wynant et al. 2017), Drosophila melanogaster encodes three Piwis (Lewis et al. 2018), but some turbellaria flatworms could have up to eight lineage specific paralogs (Fontenla et al. 2021). The varying rates of sequence evolution and gene turnover has made the animal Piwi phylogeny difficult to resolve and multiple contradicting Piwi gene trees that do not mirror species relationships have been presented (Kerner et al. 2011; Wynant et al. 2017; Fontenla et al. 2021). However, a general theme of Piwi phylogenetics suggests there are two major subfamilies, Piwil1 and Piwil2. Some trees suggested vertebrate piwis are not directly orthologous to insect Piwis (Wynant et al. 2017), others found that Drosophila Ago3 is orthologous to vertebrate Piwil2 but the Piwi and Aub genes of Drosophila do not have a vertebrate ortholog (Kerner et al. 2011; Jehn et al. 2018; Fontenla et al. 2021).

Consistent with the two subfamily generalization, vertebrate genomes encode at least two copies of the Piwi family, a copy from the Piwil1 (-iwi) group and another from the Piwil2 (-ili) group, however vertebrates have a maximum of four Piwi paralogs (See above) and questions remain about the timing and mechanism of vertebrate Piwi duplication. Here, we are interested in leveraging phylogenetic and synteny analyses to clarify the duplication history of the vertebrate Piwi family as well as understanding the patterns of selection and expression among major lineages.

\section{Methods}

Sequence acquisition

We used Ensembl v.101 (Yates et al. 2020) and NCBI release 239 (Sharma et al. 2018) to collect known Piwi and Piwi-like coding sequences (CDS) from representative species of all major groups of vertebrates. Specifically, our sampling included cyclostomes (jawless fishes; lamprey) and gnathostomes (jawed vertebrates) from chondrichthyes (cartilaginous fishes), rayfinned fishes, lobe-finned fishes, tetrapods (amphibians, reptiles, birds and mammals). To help understand the ancestral state and focus on changes that occurred within vertebrates, we included Piwis from closely related deuterostomes as outgroups (Table S1, Fig. S1). The longest CDS was selected if there were multiple transcripts for a Piwi gene. Unidentified CDSs that shared similarity to Piwis were queried against the NCBI non redundant protein database via BLAST (Altschul et al. 1990) to confirm homology. If a Piwi paralog was absent or poorly annotated in Ensembl, we queried the NCBI for the paralog using human Piwi sequences as BLAST queries.

\section{Phylogenetic reconstruction}

All Piwi sequences were translated to amino acids and aligned using the LINSI strategy in MAFFT v7 (Katoh and Standley 2013). We constructed phylogenetic trees from the amino acid

PeerJ reviewing PDF | (2021:06:62032:1:0:NEW 25 Sep 2021) 
122 alignment with two methods. First, we used IQ-Tree2 (Minh et al. 2020) and let IQ-Tree2 find

123 the best fitting substitution model (Kalyaanamoorthy et al. 2017). Ten independent phylogenetic

124 analyses were run simultaneously to explore tree space and we selected the tree with the

125 highest likelihood score. Node support for the best scoring tree was evaluated with the ultrafast

126 bootstrap method (Hoang et al. 2018) and the SH-like approximate likelihood ratio test (SH-

127 aLRT) (Guindon et al. 2010). In both cases, we used 1000 pseudoreplicates. Second, we used

128 ExaBayes v1.5.1 (Aberer et al. 2014) to conduct Bayesian analyses. We ran four simultaneous

129 chains for 1,000,000 generations sampling every 500 generations, using the same substitution

130 model chosen by IQ-Tree2 but without additional model parameters. Chains were considered as

131 having converged once the average standard deviation of split frequency among runs was less

132 than 0.01 . We summarized results with a majority-rule consensus tree from the set of sampled

133 trees after the first $25 \%$ were discarded. We repeated these tree construction analyses on a

134 reduced alignment generated after removing poorly aligned regions using Gblocks and allowed

135 up to five contiguous non-conserved positions and columns to contain gaps; -b3=5 -b5=a

136 (Castresana 2000) to test the impact of alignment quality on our results.

Synteny analyses

138

139

140

141

142

143

144

145

146

147

148

149

150

151

152

153

154

155

156

157

158

159

160

161

162

163

164

To understand the conservation of synteny as well as gene gain and loss, we examined the position of protein coding genes up and downstream of Piwi genes in representative vertebrates. We used gene coordinate information from Ensembl v.101 as well as estimates of orthology and paralogy from the Compara database (Herrero et al. 2016). If a Piwi gene was presumed missing, we used BLAST in Ensembl with human Piwi queries to locate any Piwi derived fragments near expected positions. If no fragments were found, we concluded the gene was missing, but if fragments were found and a full annotation was lacking, we presumed the Piwi to be pseudogenized.

\section{Estimating $d_{N} / d_{S}$ rates and selection tests}

For each Piwi paralog in each major lineage with multiple samples (birds, mammals, and rayfinned fishes), we made independent codon alignments by translating DNA sequences to amino acid sequences, generating LINSI amino acid alignments and 'reverse translating' alignments into codon alignments using a custom Python script (available at github.com/mike2vandy/PiwiGeneFamily along with other scripts used). We specifically conducted site-tests for diversifying selection (Goldman and Yang 1994) among alignments using the codeml module of PAML v4.9. We calculated the likelihood of models that allow $d_{N} / d_{S}$ to vary among codon positions (M1a, M2a, M8a and M8). We used the likelihood ratio test (LRT) to test for significant differences between nested models that do not allow selection to those that do (M1a vs. M2a, M8a vs. M8). We also estimated a single $d_{N} / d_{S}$ rate for each alignment using codeml's M0 one-ratio model and pairwise $d_{N} / d_{S}$ distances using the modified Nei-Gojobori (proportional) method in MEGA-X (Kumar et al. 2018). We noticed an intronic sequence that included stop codons was retained in the manatee Piwil4 sequence. We suspect this was an annotation artifact and because of it, this sequence was not included in selection tests and $d_{N} / d_{S}$ estimates. 
165

166

167

168

169

170

171

172

173

174

175

176

177

178

179

180

181

182

183

184

185

186

187

188

189

190

191

192

193

194

195

196

197

198

199

200

201

202

203

204

205

To view changes in $d_{\mathrm{N}} / d_{\mathrm{S}}$ along the mammalian Piwil1 and Piwil3 branches we constructed a phylogenetic tree of mammalian Piwil1 and Piwil3 and used a free-ratio model to estimate $d_{N} / d_{\mathrm{S}}$ rates on all branches (Yang 1998). We also used the adaptive branch-site random effects likelihood (aBSREL; (Smith et al. 2015)) approach implemented in Datamonkey v2.2 (Weaver et al. 2018) which detects episodes of positive selection on all designated foreground branches.

We performed a LRT between the null model $\left(d_{N} / d_{S}=1\right)$ against the alternative where a branch was undergoing some form of selection $\left(d_{N} / d_{\mathrm{S}} \neq 1\right)$. All Piwil3 branches were labeled as foreground and tested.

Expression analyses

To assess historical changes in Piwi expression through vertebrate evolution, we collected RNASeq data from representative tissues (brain, heart, kidney, liver, ovary and testes) and species (elephant shark, spotted gar, clawed frog, spiny toad, chicken, opossum, cow, and human) from NCBI's short read archive (SRA; (Leinonen et al. 2011). Accession numbers for species and tissues can be found in Table S2. For all species, we used appropriate Ensembl's CDSs downloaded from BioMart as a mapping reference. We only used the longest CDS per gene. Prior to mapping, RNASeq reads were cleaned with Trimmomatic v0.38 (Bolger et al. 2014) using the trimming and adapter removal parameters: HEADCROP:5, SLIDINGWINDOW:5:30, MINLEN:50, and ILLUMINACLIP:2:30:10. RNASeq reads were mapped to reference CDS libraries using default parameters of RSEM v1.3.1 (Li and Dewey 2011) and Bowtie v1.2.2 (Langmead et al. 2009) to estimate gene expression in units of transcripts per million (TPM).

\section{Results}

Phylogenetic analysis

Our first goal was to reconstruct the Piwi phylogeny from vertebrate representatives and deuterostome outgroups using both maximum likelihood (ML) and Bayesian strategies. We collected 168 sequences from 63 species (Table S1). We generated trees in three different strategies: a tree of select representative species (Fig. 1), a tree from all collected sequences (Fig. S2), and because the $\mathrm{N}$-terminus was difficult to align, we also estimated a tree from the most alignable regions of the amino acid alignment which included the PAZ and PIWI domains (Fig S3). All trees were relatively similar and sequences fell into the two major clades, the Piwil1 group and Piwil2 group (Fig. 1). There have been no additional duplications of Piwil2 among deuterostomes and the subfamily is monophyletic. By contrast, Piwil4 and the mammalian specific Piwil3 were derived from independent duplications of a vertebrate Piwil1 (Fig. 1). These additional duplications within the Piwil1 clade indicate that the gnathostome Piwil1 is not a 1:1 ortholog of the ancestral Piwil1 gene. However, we have retained the traditional nomenclature for simplicity.

The Piwil4 gene of vertebrates was likely derived from an ancient duplication of Piwil1 in the ancestor of gnathostomes, however a lamprey Piwil1-like sequence was found sister to gnathostome Piwil4 in both ML and Bayesian trees. Therefore, we can interpret the Piwil4 
206

207

208

209

210

211

212

213

214

215

216

217

218

219

220

221

222

223

224

225

226

227

228

229

230

231

232

233

234

235

236

237

238

239

240

241

242

243

244

245

duplication in two different ways. The Piwil4 duplication occurred in the ancestor of vertebrates, but Piwil1 was lost in lamprey and the lamprey sequence is an ortholog of Piwil4. Alternatively, the Piwil4 duplication occurred in gnathostomes, but the lamprey Piwil1-like gene is artificially sister to Piwil4. Piwil4 was present in cartilaginous and lobe-finned fishes, mammals and reptiles, but was absent in ray-finned fishes and birds (Fig. 1; Fig. S1).

Our analyses suggest Piwil3 is the result of a duplication of Piwil1 in the common ancestor of therian mammals given its presence in marsupial and placental mammals and its absence from Monotreme genomes (Fig. 1). However, not all placental mammals have retained Piwil3. Piwil3 was present in marsupials (opossum and koala), Laurasiatherians (bat, dog, and cow) and most Euarchontoglires (primates, rodents and relatives), but we were unable to find it in Afrotherians (elephant and manatee), Xenarthrans (armadillo) and mouse-like rodents which included the kangaroo rat, deer mouse and house mouse (Fig. S1).

An additional copy of Piwil1 (Piwil1a and Piwil1b) had been previously described in the clawed frog (Wilczynska et al. 2009), but our analyses also revealed this additional paralog was present in the spiny toad (Fig. S2). Interestingly, a third intact Piwil1 copy was identified in the spiny toad (Fig. S2). This copy is most likely a retrogene, diagnosed by a lack of introns in the coding sequence (Fig. S4A).

Synteny analyses

We used genomic coordinates to identify genes up- and down-stream of Piwi genes to resolve or confirm homology and infer the duplication mechanism behind the vertebrate Piwi expansion. Synteny analyses included representative species with contiguous regions for at least 10 genes up and downstream of each Piwi. In most cases, this included the elephant shark, spotted gar, zebra fish, clawed frog and chicken and human, but varied among Piwis. The lamprey assembly on Ensembl lacked contiguity around the Piwi genes, therefore we queried sea lamprey coordinate data from a GFF file at https://genomes.stowers.org/sealamprey (Smith et al. 2018). Unfortunately, there was a lack of synteny among Piwi genes between cyclostomes and gnathostomes, but synteny around Piwil1, Piwil2 and Piwil4 has generally been conserved among gnathostomes (Fig. 2; Fig. S5). Synteny around Piwil2 was less conserved than Piwil1 and observed three different sets of upstream genes, however Slc39a14 and Ppp3cc were consistently downstream of Piwil2 in all lineages (Fig. S5).

Piwil1 was flanked by Rimbp2, Stx2, and Ran on the 3' end in all gnathostomes, but synteny was only conserved upstream of Piwil1 among bony and jawed vertebrates where Piwil1 is generally flanked by Fzd10, Tmem132d and Glt1d1 (Fig. 2A). By contrast, synteny around Piwil4 has been conserved throughout all gnathostome evolution. Although Piwil4 was lost in ray-finned fish and birds, the overall locus has remained intact (Fig. 2B). In the spotted gar, the distance between Fut4 and Amotl1 was 18,372 bases and there were no BLAST hits to Piwi genes in that region. The same was true for the chicken, where the distance between Fut4 and Amotl1 was 45,906 and lacked sequences similar to Piwil4. By contrast, the same region in the crocodile covers 157,135 bases and includes a Piwil4 gene (Fig. 2B). Interestingly, Piwil4 is intact in all mammals except the platypus. The Piwil4 locus in platypus does contain a IncRNA

Peer) reviewing PDF | (2021:06:62032:1:0:NEW 25 Sep 2021) 
246

247

248

249

250

251

252

253

254

255

256

257

258

259

260

261

262

263

264

265

266

267

268

269

270

271

272

273

274

275

276

277

278

279

280

281

282

annotation (ENSOANT00000069592) between Fut4 and Amotl1 with similarity to Piwil4. However, a complete gene with an open reading frame could not be recovered. Therefore we suspect that Piwil4 has been pseudogenized in the platypus (Fig. 2B) but has not been fully purged from the genome.

Piwil1 and Piwil4 were not flanked by any co-duplicating gene families (Fig. 2), an indicative signal of whole genome or segmental duplications (Campanini et al. 2015; Opazo et al. 2015). Furthermore, both teleosts and salmonid fishes, which have experienced additional whole genome duplication events (Jaillon et al. 2004; Macqueen and Johnston 2014; Lien et al. 2016), lacked any additional Piwi paralogs (Fig. S2).

Piwil3 is a direct neighbor of Piwil1 in marsupials which would suggest that the Piwil1 and Piwil3 genes of therian mammals are co-orthologs of the ancestral mammalian Piwil1 gene derived from a tandem duplication of Piwil1 in their common ancestor (Fig. 3A). Interestingly, the position of Piwil3 is conserved among marsupials, but Piwil 3 is in a novel location in placental mammals where flanking genes vary among lineages (Fig. 3A). Taking these results together, the expansion of the Piwi gene family in vertebrates can best be explained by tandem duplication followed by a translocation due to lineage-specific genomic rearrangements.

\section{Piwil3 and selection tests}

We observed very long branches among Piwil3 paralogs (Fig. 1) and decided to test the role diversifying selection has played on the evolution of Piwil3. Using PAML's free ratio model, we found that $d_{N} / d_{S}$ rates among Piwil3 sequences were considerably faster than rates among therian Piwil1 sequences (Fig. 3B). Since the free-ratio model does not offer any additional information besides changes in $d_{N} / d_{S}$ and is not statistically robust, we used site-tests among Piwil3 sequences and found that models allowing selection (M2a and M8) were significantly better fits to the data than models that disallow positive selection (M1a and M8a) (Table S3). Codons throughout the entire gene, including the conserved PAZ and PIWI domains (Fig. S6), exhibited signatures of positive selection (Fig. 3C; Fig. S7) according to Bayes Empirical Bayes (BEB) estimation of site posterior probabilities. A separate branch-site test, aBSREL in HyPhy, suggested 17 out of 22 tested Piwil3 branches have undergone episodic positive selection (Fig. S8).

We then compared Piwil3 patterns of evolution to the other paralogs within tetrapod groups (birds, mammals and ray-finned fishes). Site-tests of diversifying selection suggested no other Piwi paralog is evolving in a diversifying pattern. In all cases except Piwil3, models allowing positive selection were not improved over models restricting selection (Table S3). Further, oneratio (Fig. 3D) and pairwise (Fig. $3 \mathrm{E}) d_{N} / d_{\mathrm{S}}$, although likely inflated due to saturation at synonymous sites, indicated Piwil3 is the fastest evolving Piwi among all lineages while Piwil1 in mammals is highly conserved.

Historical Piwi expression 
283

284

285

286

287

288

289

290

291

292

293

294

295

296

297

298

299

300

301

302

303

304

305

306

307

308

309

310

311

312

313

314

315

316

317

318

319

320

321

322

323

Our last goal was to identify any differences in Piwi expression among lineages, and consistent with previous results, Piwi expression was largely localized to the gonads, specifically testis, although Piwis were sporadically expressed at low levels among somatic tissues (Fig. 4A). Aravin et al. (2008) reported that Piwil4 is only expressed for a short period of time during testis development in mice, but Piwil4 has since been detected in adult undifferentiated spermatogonia (Carrieri et al. 2017; Vasiliauskaitè et al. 2018) Consistently, Piwil4 was detected in the adult testes of all species examined, but lowly detected in the mouse (Fig. 4B). In addition, we detected weak expression of Piwil3 in the testis of the opossum, human, and cattle. Both Piwil1 paralogs were expressed in the testis of the clawed frog (Fig. 4B). We were also able to measure Piwi expression from the brain, ovary, and testes, in the spiny toad which had an additional Piwil1 retrogene. The spiny toad expression pattern was unique as Piwil4 and Piwil1a-retro were the only Piwis expressed in the testis, but Piwil1a and Piwil1a-retro were expressed in the ovary (Fig S4B). Piwil1b nor Piwil2 were expressed in any library (Fig. S4B).

\section{Discussion}

Understanding the relationships among gene family members is an active area of research in evolutionary biology and the availability of whole-genome sequences allows the opportunity to address unresolved questions. The Piwi gene family is a charismatic family of proteins that function as a genome defense mechanism against invasive elements that include TEs and viruses and function in a similar fashion as CRiSPR/Cas9. However, the paralog relationships and duplication timing in vertebrates has not been thoroughly resolved.

Consistent with previous work, Piwil2 was found to be monophyletic among deuterostomes (Kerner et al. 2011), but the Piwil1 group has experienced additional duplications among vertebrates giving rise to the gnathostome Piwil4 and the therian Piwil3 paralogs (Fig. 1). Based on our phylogenetic and synteny analyses, we propose a model where Piwil4 originated from the duplication of Piwil1 early in vertebrate evolutionary history, at least as early as the ancestor of gnathostomes (Fig. 5). Unfortunately, the exact timing of Piwil4 origination is unresolved given that orthology could not be accurately inferred between cyclostomes and gnathostomes. Cyclostome genomes possess nucleotide biases and are the product of an additional cyclostome specific whole-genome triplication (Nakatani et al. 2021) and resolving orthology between gnathostome and cyclostome genes is often difficult (Qiu et al. 2011; Kuraku 2013; Smith et al. 2013; Campanini et al. 2015; Opazo et al. 2015). However, given that 1- Piwil4 is secondarily lost, 2- all vertebrates have a Piwil1, and 3- there are only two Piwi paralogs in the lamprey genome, we propose that Piwil4 probably emerged in the gnathostome lineage, after the divergence between gnathostomes and cyclostomes. Piwil4 was then secondarily lost in the common ancestor of ray-finned fishes, retained in lobe-finned fishes, Sarcopterygii, and its descendants, but lost again in birds and monotremes (Fig. 5). Piwil3 originated in the common ancestor marsupial and placental mammals as a tandem duplication, but the retention and position of Piwil3 is variable among placental mammals (Fig. 3A).

Piwil3 was not identified in the elephant or armadillo genome. Under the most parsimonious scenario, Xenarthra and Afrotheria belong to the monophyletic group Atlantogenata (Foley et al. 2016), and Piwil3 was lost in the common ancestor (Fig. S1). In addition, Piwil3 was also

Peer) reviewing PDF | (2021:06:62032:1:0:NEW 25 Sep 2021) 
324

325

326

327

328

329

330

331

332

333

334

335

336

337

338

339

340

341

342

343

344

345

346

347

348

349

350

351

352

353

354

355

356

357

358

359

360

361

362

363

364

365

secondarily lost in some mouse-like rodents. Among Glires, rabbits, guinea pigs and beavers have retained Piwil3 (Fig. 2B), while the deer mouse, kangaroo rat, and house mouse lost Piwil3. The beaver is nested within the mouse-like rodent clade that includes the deer mouse, house mouse, and kangaroo rat (Blanga-Kanfi et al. 2009; Fabre et al. 2012), and encodes a Piwil3 while the remaining three species lack Piwil3 (Fig. 2B). This points to a complex scenario of gene retention (See Fig. S1) that would require further investigation to unveil.

We performed synteny analyses in an attempt to identify the duplication mechanism, be it whole genome, segmental, or tandem duplication. Piwil1 and Piwil2 were already present in the ancestor of deuterostomes so these paralogs are the product of a duplication early in animal evolution (Kerner et al. 2011), and we could not detect any co-duplicating gene families neighboring Piwil1 or Piwil4 (Fig. 2) that would point to segmental or whole genome duplication (Catchen et al. 2009). In addition, Piwil3 is a direct neighbor of Piwil1 in the opossum genome, which likely reflects the ancestral state. Piwil3 likely migrated to a novel locus through nonhomologous recombination in the ancestor of placental mammals, and synteny has not been conserved (Fig. 3A). From this evidence we conclude tandem duplication of Piwil1 followed by non-homologous recombination drove the expansion of the Piwi gene family.

The additional duplication events within the Piwil1 group likely led to novel functions among paralogs. PIWI proteins in mice bind piRNAs of different sizes and knockouts among paralogs yield discrete phenotypes (Aravin et al. 2007; Aravin et al. 2008, Kuramochi-Miyagawa et al. 2008). Unfortunately the exact function of all paralogs in all major lineages is unknown, but expression initiation varies among lineages which points to slightly different functions. For example, Piwil1 expression begins in primordial germ cells at E7 in zebrafish (Hsu et al. 2018) and E6 in chicken (Kim et al. 2012), but Piwil1 expression begins during meiosis at P14 in mice (Girard et al. 2006). Results from our $d_{N} / d_{S}$ analyses suggest differences in selective pressure that could be related to these functional differences. Given the expression and $d_{N} / d_{S}$ differences, we can hypothesize that the function of PIWIL1 removing mRNAs in later stages of meiosis could be unique to mammals. We also hypothesize there may be some functional redundancy between PIWIL2 and PIWIL4. Because PIWIL4 localizes to the nucleus and PIWIL2 localizes to the cytoplasm, it is thought that PIWIL2 is responsible for loading PIWIL4 with secondary piRNAs and PIWIL4 "marks" TE loci for subsequent methylation (Carmell et al. 2007; Aravin et al. 2008). Interestingly, there is some evidence that both PIWIL2 and PIWIL4 function to silence TE expression through methylation pathways (Manakov et al. 2015), although this claim is contested in Zoch et al. (2020). However, PIWIL2 does localize to the nucleus in zebrafish (Houwing et al. 2008) and although untested, could potentially play a role in TE methylation (Goll and Halpern 2011). Since both ray-finned fishes, birds lack Piwil4, it would be of interest to determine if the lack of Piwil 4 has resulted in any measurable effect on TE methylation or test a methylation initiation function for PIWIL2 in these lineages.

Selection tests revealed evidence that Piwil3 is evolving in a fast and diversifying manner in all mammalian lineages (Fig. 3B; Fig. S8) and no Piwi in any other tested lineage is evolving in a diversifying manner (Table S3). The fast rate of Piwil 3 was previously revealed in simian primates (Wynant et al. 2017), but we demonstrated this phenomenon is not exclusive to primates. It is unclear why Piwil3 would exhibit an increased rate of evolution. Genes that tend

Peer) reviewing PDF | (2021:06:62032:1:0:NEW 25 Sep 2021) 
366

367

368

369

370

371

372

373

374

375

376

377

378

379

380

381

382

383

384

385

386

387

388

389

390

391

392

393

394

395

396

397

398

399

400

401

402

403

404

405

to evolve in this fashion are often associated with immunity/defense against pathogens and reproduction/sexual selection (Kosiol et al. 2008; Park et al. 2011; Vandewege et al. 2013; Grueber et al. 2014; Vandewege et al. 2020). Further, RNAi/antiviral genes have higher rates of evolution in insects relative to other genes (Obbard et al. 2009; Wynant et al. 2017). Piwil1 and Piwil2, among other piRNA pathway genes, have high $d_{N} / d_{S}$ rates and appear to be evolving under positive selection in teleost fishes that have wide TE diversity (Yi et al. 2014). Since Piwil3 is possibly part of a genome immune system, it could be plausible Piwil3 adapts to lineage specific genome stressors. However, Piwil3 is not present in all mammalian lineages and mutant knockout experiments have not been conducted to understand its precise function, so there is currently no good explanation as to why Piwil3 is evolving so fast.

In general, our expression results were consistent with the literature. Piwis exhibited the highest expression in germline tissues (Fig. 4A) and Piwil1 and Piwil2 were the most expressed in adult testis (Fig. 4B). We also detected the expression of Piwil4 in adult testis among all gnathostomes that encode a Piwil4, albeit expression was lowest in the mouse (Fig. 4B). In mice, Piwil4 expression coincides with gonocyte methylation erasure and re-establishment (Aravin et al. 2008; Carmell 2008; Molaro et al. 2014) where the function is linked to homeostatic and regenerative spermatogenesis (Carrieri et al. 2017; Vasiliauskaitè et al. 2018). There were some inconsistencies regarding ovarian Piwi expression. Piwi expression has been previously detected in the clawed frog (Wilczynska et al. 2009) zebrafish (Houwing et al. 2007; Houwing et al. 2008), humans and cattle. (Roovers et al. 2015). Piwil3 has only been described in maturing oocytes and early embryos (Tan et al. 2020) but we found it expressed in testis (Fig. 4A) although it has not been described in testis. Nonetheless, all four Piwis were expressed in both opossum gonads suggesting Piwil3 localization to gonads was already established in the common ancestor of therian mammals.

\section{Conclusions}

We present an evolutionary study of the Piwi gene family in vertebrates that is generally composed of two to four paralogs with emphasis on resolving the phylogenetic relationships and duplication history. Our study included representative species from all of the major groups of vertebrates and deuterostome outgroups. Piwil2 has not undergone any additional duplications through vertebrate evolution, but Piwil4 and Piwil3 are derived from independent duplications of the vertebrate Piwil1 at different times. Piwil4 likely first appeared in the ancestor of gnathostomes, but was secondarily lost in ray-finned fishes and birds, and Piwil3 is therian specific. The most likely mechanism yielding these novel paralogs was tandem duplication followed by non-homologous recombination. Piwi genes are largely evolving at different rates among lineages and expression has largely been constrained to the gonads through all of vertebrate history.

\section{Acknowledgements}

The color palette was inspired by Prince and the Revolution's Purple Rain (1984).

\section{References}

Peer] reviewing PDF | (2021:06:62032:1:0:NEW 25 Sep 2021) 
406

407

408

409

410

411

412

413

414

415

416

417

418

419

420

421

422

423

424

425

426

427

428

429

430

431

432

433

434

435

436

437

438

439

440

441

442

443

Aberer AJ, Kobert K, Stamatakis A. 2014. ExaBayes: massively parallel bayesian tree inference for the whole-genome era. Mol. Biol. Evol. 31:2553-2556.

Altschul SF, Gish W, Miller W, Myers EW, Lipman DJ. 1990. Basic local alignment search tool. J. Mol. Biol. 215:403-410.

Aravin AA, Sachidanandam R, Bourc'his D, Schaefer C, Pezic D, Toth KF, Bestor T, Hannon GJ. 2008. A piRNA pathway primed by individual transposons is linked to de novo DNA methylation in mice. Mol. Cell 31:785-799.

Aravin AA, Sachidanandam R, Girard A, Fejes-Toth K, Hannon GJ. 2007. Developmentally regulated piRNA clusters implicate MILI in transposon control. Science 316:744-747.

Blanga-Kanfi S, Miranda H, Penn O, Pupko T, DeBry RW, Huchon D. 2009. Rodent phylogeny revised: analysis of six nuclear genes from all major rodent clades. BMC Evol. Biol. 9:71.

Bolger AM, Lohse M, Usadel B. 2014. Trimmomatic: a flexible trimmer for Illumina sequence data. Bioinformatics 30:2114-2120.

Brennecke J, Aravin AA, Stark A, Dus M, Kellis M, Sachidanandam R, Hannon GJ. 2007. Discrete small RNA-generating loci as master regulators of transposon activity in Drosophila. Cell 128:1089-1103.

Campanini EB, Vandewege MW, Pillai NE, Tay B-H, Jones JL, Venkatesh B, Hoffmann FG. 2015. Early Evolution of Vertebrate Mybs: An Integrative Perspective Combining Synteny, Phylogenetic, and Gene Expression Analyses. Genome Biol. Evol. 7:3009-3021.

Carmell M. 2008. PIWI Proteins Are Essential for Spermatogenesis and Repression of Transposons in the Mouse Male Germline. Biology of Reproduction [Internet] 78:160-160. Available from: http://dx.doi.org/10.1093/biolreprod/78.s1.160a

Carmell MA, Girard A, van de Kant HJG, Bourc'his D, Bestor TH, de Rooij DG, Hannon GJ. 2007. MIWI2 Is Essential for Spermatogenesis and Repression of Transposons in the Mouse Male Germline. Developmental Cell [Internet] 12:503-514. Available from: http://dx.doi.org/10.1016/j.devcel.2007.03.001

Carmell MA, Xuan Z, Zhang MQ, Hannon GJ. 2002. The Argonaute family: tentacles that reach into RNAi, developmental control, stem cell maintenance, and tumorigenesis. Genes Dev. 16:2733-2742.

Carrieri C, Comazzetto S, Grover A, Morgan M, Buness A, Nerlov C, O'Carroll D. 2017. A transit-amplifying population underpins the efficient regenerative capacity of the testis. $\mathrm{J}$. Exp. Med. 214:1631-1641.

Castresana J. 2000. Selection of conserved blocks from multiple alignments for their use in phylogenetic analysis. Mol. Biol. Evol. 17:540-552.

Catchen JM, Conery JS, Postlethwait JH. 2009. Automated identification of conserved synteny after whole-genome duplication. Genome Res. 19:1497-1505.

Fabre P-H, Hautier L, Dimitrov D, Douzery EJP. 2012. A glimpse on the pattern of rodent diversification: a phylogenetic approach. BMC Evol. Biol. 12:88. 
444

445

446

447

448

449

450

451

452

453

454

455

456

457

458

459

460

461

462

463

464

465

466

467

468

469

470

471

472

473

474

475

476

477

478

479

480

481
Foley NM, Springer MS, Teeling EC. 2016. Mammal madness: is the mammal tree of life not yet resolved? Philos. Trans. R. Soc. Lond. B Biol. Sci. [Internet] 371. Available from: http://dx.doi.org/10.1098/rstb.2015.0140

Fontenla S, Rinaldi G, Tort JF. 2021. Lost and Found: Piwi and Argonaute Pathways in Flatworms. Front. Cell. Infect. Microbiol. 11:653695.

Girard A, Sachidanandam R, Hannon GJ, Carmell MA. 2006. A germline-specific class of small RNAs binds mammalian Piwi proteins. Nature 442:199-202.

Goldman N, Yang Z. 1994. A codon-based model of nucleotide substitution for protein-coding DNA sequences. Mol. Biol. Evol. 11:725-736.

Goll MG, Halpern ME. 2011. DNA Methylation in Zebrafish. Progress in Molecular Biology and Translational Science [Internet]:193-218. Available from: http://dx.doi.org/10.1016/b978-012-387685-0.00005-6

Gou L-T, Dai P, Yang J-H, Xue Y, Hu Y-P, Zhou Y, Kang J-Y, Wang X, Li H, Hua M-M, et al. 2015. Pachytene piRNAs instruct massive mRNA elimination during late spermiogenesis. Cell Research [Internet] 25:266-266. Available from: http://dx.doi.org/10.1038/cr.2015.14

Grueber CE, Wallis GP, Jamieson IG. 2014. Episodic positive selection in the evolution of avian toll-like receptor innate immunity genes. PLoS One 9:e89632.

Guindon S, Dufayard J-F, Lefort V, Anisimova M, Hordijk W, Gascuel O. 2010. New algorithms and methods to estimate maximum-likelihood phylogenies: assessing the performance of PhyML 3.0. Syst. Biol. 59:307-321.

Han BW, Wang W, Li C, Weng Z, Zamore PD. 2015. piRNA-guided transposon cleavage initiates Zucchini-dependent, phased piRNA production. Science [Internet] 348:817-821. Available from: http://dx.doi.org/10.1126/science.aaa1264

Herrero J, Muffato M, Beal K, Fitzgerald S, Gordon L, Pignatelli M, Vilella AJ, Searle SMJ, Amode R, Brent S, et al. 2016. Ensembl comparative genomics resources. Database [Internet] 2016. Available from: http://dx.doi.org/10.1093/database/baw053

Hoang DT, Chernomor O, von Haeseler A, Minh BQ, Vinh LS. 2018. UFBoot2: Improving the Ultrafast Bootstrap Approximation. Mol. Biol. Evol. 35:518-522.

Houwing S, Berezikov E, Ketting RF. 2008. Zili is required for germ cell differentiation and meiosis in zebrafish. EMBO J. 27:2702-2711.

Houwing S, Kamminga LM, Berezikov E, Cronembold D, Girard A, van den Elst H, Filippov DV, Blaser H, Raz E, Moens CB, et al. 2007. A role for Piwi and piRNAs in germ cell maintenance and transposon silencing in Zebrafish. Cell 129:69-82.

Hsu C-W, Pan Y-J, Wang Y-W, Tong S-K, Chung B-C. 2018. Changes in the morphology and gene expression of developing zebrafish gonads. Gen. Comp. Endocrinol. 265:154-159.

Jaillon O, Aury J-M, Brunet F, Petit J-L, Stange-Thomann N, Mauceli E, Bouneau L, Fischer C, Ozouf-Costaz C, Bernot A, et al. 2004. Genome duplication in the teleost fish Tetraodon nigroviridis reveals the early vertebrate proto-karyotype. Nature 431:946-957.

Peer) reviewing PDF | (2021:06:62032:1:0:NEW 25 Sep 2021) 
482

483

484

485

486

487

488

489

490

491

492

493

494

495

496

497

498

499

500

501

502

503

504

505

506

507

508

509

510

511

512

513

514

515

516

517

518

519

Jehn J, Gebert D, Pipilescu F, Stern S, Kiefer JST, Hewel C, Rosenkranz D. 2018. genes and piRNAs are ubiquitously expressed in mollusks and show patterns of lineage-specific adaptation. Commun Biol 1:137.

Kolliopoulou A, Santos D, Taning CNT, Wynant N, Vanden Broeck J, Smagghe G, Swevers L. 2019. PIWI pathway against viruses in insects. Wiley Interdiscip. Rev.: RNA 2019, e1555

Kalyaanamoorthy S, Minh BQ, Wong TKF, von Haeseler A, Jermiin LS. 2017. ModelFinder: fast model selection for accurate phylogenetic estimates. Nat. Methods 14:587-589.

Katoh K, Standley DM. 2013. MAFFT multiple sequence alignment software version 7: improvements in performance and usability. Mol. Biol. Evol. 30:772-780.

Kerner P, Degnan SM, Marchand L, Degnan BM, Vervoort M. 2011. Evolution of RNA-binding proteins in animals: insights from genome-wide analysis in the sponge Amphimedon queenslandica. Mol. Biol. Evol. 28:2289-2303.

Kim TH, Yun TW, Rengaraj D, Lee SI, Lim SM, Seo HW, Park TS, Han JY. 2012. Conserved functional characteristics of the PIWI family members in chicken germ cell lineage. Theriogenology 78:1948-1959.

Kolliopoulou A, Santos D, Taning CNT, Wynant N, Vanden Broeck J, Smagghe G, Swevers L. 2019. PIWI pathway against viruses in insects. Wiley Interdiscip. Rev. RNA 10:e1555.

Kosiol C, Vinar T, da Fonseca RR, Hubisz MJ, Bustamante CD, Nielsen R, Siepel A. 2008. Patterns of positive selection in six Mammalian genomes. PLoS Genet. 4:e1000144.

Kumar S, Stecher G, Li M, Knyaz C, Tamura K. 2018. MEGA X: Molecular Evolutionary Genetics Analysis across Computing Platforms. Mol. Biol. Evol. 35:1547-1549.

Kumar S, Stecher G, Suleski M, Hedges SB. 2017. TimeTree: A Resource for Timelines, Timetrees, and Divergence Times. Mol. Biol. Evol. 34:1812-1819.

Kuraku S. 2013. Impact of asymmetric gene repertoire between cyclostomes and gnathostomes. Semin. Cell Dev. Biol. 24:119-127.

Kuramochi-Miyagawa S, Watanabe T, Gotoh K, Totoki Y, Toyoda A, Ikawa M, Asada N, Kojima $\mathrm{K}$, Yamaguchi $\mathrm{Y}$, ljiri TW, et al. 2008. DNA methylation of retrotransposon genes is regulated by Piwi family members MILI and MIWI2 in murine fetal testes. Genes \& Development [Internet] 22:908-917. Available from: http://dx.doi.org/10.1101/gad.1640708

Langmead B, Trapnell C, Pop M, Salzberg SL. 2009. Ultrafast and memory-efficient alignment of short DNA sequences to the human genome. Genome Biol. 10:R25.

Lau NC, Seto AG, Kim J, Kuramochi-Miyagawa S, Nakano T, Bartel DP, Kingston RE. 2006. Characterization of the piRNA complex from rat testes. Science 313:363-367.

Leinonen R, Sugawara H, Shumway M, International Nucleotide Sequence Database Collaboration. 2011. The sequence read archive. Nucleic Acids Res. 39:D19-D21.

Lewis SH, Quarles KA, Yang Y, Tanguy M, Frézal L, Smith SA, Sharma PP, Cordaux R, Gilbert C, Giraud I, et al. 2018. Pan-arthropod analysis reveals somatic piRNAs as an ancestral defence against transposable elements. Nat Ecol Evol 2:174-181.

PeerJ reviewing PDF | (2021:06:62032:1:0:NEW 25 Sep 2021) 
520

521

522

523

524

525

526

527

528

529

530

531

532

533

534

535

536

537

538

539

540

541

542

543

544

545

546

547

548

549

550

551

552

553

554

555

556

557

558

Li B, Dewey CN. 2011. RSEM: accurate transcript quantification from RNA-Seq data with or without a reference genome. BMC Bioinformatics 12:323.

Lien S, Koop BF, Sandve SR, Miller JR, Kent MP, Nome T, Hvidsten TR, Leong JS, Minkley DR, Zimin A, et al. 2016. The Atlantic salmon genome provides insights into rediploidization. Nature 533:200-205.

Li XZ, Roy CK, Dong X, Bolcun-Filas E, Wang J, Han BW, Xu J, Moore MJ, Schimenti JC, Weng Z, et al. 2013. An ancient transcription factor initiates the burst of piRNA production during early meiosis in mouse testes. Mol. Cell 50:67-81.

Macqueen DJ, Johnston IA. 2014. A well-constrained estimate for the timing of the salmonid whole genome duplication reveals major decoupling from species diversification. Proc. Biol. Sci. 281:20132881.

Manakov SA, Pezic D, Marinov GK, Pastor WA, Sachidanandam R, Aravin AA. 2015. MIWI2 and MILI Have Differential Effects on piRNA Biogenesis and DNA Methylation. Cell Rep. 12:1234-1243.

Minh BQ, Schmidt HA, Chernomor O, Schrempf D, Woodhams MD, von Haeseler A, Lanfear R. 2020. IQ-TREE 2: New Models and Efficient Methods for Phylogenetic Inference in the Genomic Era. Mol. Biol. Evol. 37:1530-1534.

Mohn F, Handler D, Brennecke J. 2015. Noncoding RNA. piRNA-guided slicing specifies transcripts for Zucchini-dependent, phased piRNA biogenesis. Science 348:812-817.

Molaro A, Falciatori I, Hodges E, Aravin AA, Marran K, Rafii S, McCombie WR, Smith AD, Hannon GJ. 2014. Two waves of de novo methylation during mouse germ cell development. Genes Dev. 28:1544-1549.

Nakatani Y, Shingate P, Ravi V, Pillai NE, Prasad A, McLysaght A, Venkatesh B. 2021. Reconstruction of proto-vertebrate, proto-cyclostome and proto-gnathostome genomes provides new insights into early vertebrate evolution. Nat. Commun. 12:4489.

Obbard DJ, Gordon KHJ, Buck AH, Jiggins FM. 2009. The evolution of RNAi as a defence against viruses and transposable elements. Philos. Trans. R. Soc. Lond. B Biol. Sci. 364:99-115.

Opazo JC, Lee AP, Hoffmann FG, Toloza-Villalobos J, Burmester T, Venkatesh B, Storz JF. 2015. Ancient Duplications and Expression Divergence in the Globin Gene Superfamily of Vertebrates: Insights from the Elephant Shark Genome and Transcriptome. Mol. Biol. Evol. 32:1684-1694.

Ozata DM, Gainetdinov I, Zoch A, O'Carroll D, Zamore PD. 2019. PIWI-interacting RNAs: small RNAs with big functions. Nat. Rev. Genet. 20:89-108.

Park SH, Podlaha O, Grus WE, Zhang J. 2011. The microevolution of V1r vomeronasal receptor genes in mice. Genome Biol. Evol. 3:401-412.

Qiu H, Hildebrand F, Kuraku S, Meyer A. 2011. Unresolved orthology and peculiar coding sequence properties of lamprey genes: the KCNA gene family as test case. BMC Genomics 12:325.

Peer) reviewing PDF | (2021:06:62032:1:0:NEW 25 Sep 2021) 
559

560

561

562

563

564

565

566

567

568

569

570

571

572

573

574

575

576

577

578

579

580

581

582

583

584

585

586

587

588

589

590

591

592

593

594

595

596

597

Reuter M, Berninger P, Chuma S, Shah H, Hosokawa M, Funaya C, Antony C, Sachidanandam R, Pillai RS. 2011. Miwi catalysis is required for piRNA amplification-independent LINE1 transposon silencing. Nature [Internet] 480:264-267. Available from: http://dx.doi.org/10.1038/nature10672

Roovers EF, Rosenkranz D, Mahdipour M, Han C-T, He N, Chuva de Sousa Lopes SM, van der Westerlaken LAJ, Zischler H, Butter F, Roelen BAJ, et al. 2015. Piwi proteins and piRNAs in mammalian oocytes and early embryos. Cell Rep. 10:2069-2082.

Rozhkov NV, Hammell M, Hannon GJ. 2013. Multiple roles for Piwi in silencing Drosophila transposons. Genes Dev. 27:400-412.

Sharma S, Ciufo S, Starchenko E, Darji D, Chlumsky L, Karsch-Mizrachi I, Schoch CL. 2018. The NCBI BioCollections Database. Database [Internet] 2018. Available from: http://dx.doi.org/10.1093/database/bay006

Siomi MC, Sato K, Pezic D, Aravin AA. 2011. PIWI-interacting small RNAs: the vanguard of genome defence. Nat. Rev. Mol. Cell Biol. 12:246-258.

Smith JJ, Kuraku S, Holt C, Sauka-Spengler T, Jiang N, Campbell MS, Yandell MD, Manousaki T, Meyer A, Bloom OE, et al. 2013. Sequencing of the sea lamprey (Petromyzon marinus) genome provides insights into vertebrate evolution. Nat. Genet. 45:415-421, 421e1-e2.

Smith JJ, Timoshevskaya N, Ye C, Holt C, Keinath MC, Parker HJ, Cook ME, Hess JE, Narum SR, Lamanna F, et al. 2018. The sea lamprey germline genome provides insights into programmed genome rearrangement and vertebrate evolution. Nat. Genet. 50:270-277.

Smith MD, Wertheim JO, Weaver S, Murrell B, Scheffler K, Kosakovsky Pond SL. 2015. Less is more: an adaptive branch-site random effects model for efficient detection of episodic diversifying selection. Mol. Biol. Evol. 32:1342-1353.

Sun YH, Xie LH, Zhuo X, Chen Q, Ghoneim D, Zhang B, Jagne J, Yang C, Li XZ. 2017. Domestic chickens activate a piRNA defense against avian leukosis virus. Elife [Internet] 6. Available from: http://dx.doi.org/10.7554/eLife.24695

Tang G. 2005. siRNA and miRNA: an insight into RISCs. Trends in Biochemical Sciences [Internet] 30:106-114. Available from: http://dx.doi.org/10.1016/j.tibs.2004.12.007

Tan M, van Tol HTA, Rosenkranz D, Roovers EF, Damen MJ, Stout TAE, Wu W, Roelen BAJ. 2020. PIWIL3 Forms a Complex with TDRKH in Mammalian Oocytes. Cells [Internet] 9. Available from: http://dx.doi.org/10.3390/cells9061356

Vandewege MW, Phillips CJ, Wickliffe JK, Hoffmann FG. 2013. Evolution of the ABPA subunit of androgen-binding protein expressed in the submaxillary glands in New and Old World rodent taxa. J. Mol. Evol. 76:324-331.

Vandewege MW, Sotero-Caio CG, Phillips CD. 2020. Positive Selection and Gene Expression Analyses from Salivary Glands Reveal Discrete Adaptations within the Ecologically Diverse Bat Family Phyllostomidae. Genome Biol. Evol. 12:1419-1428.

Vasiliauskaitė L, Berrens RV, Ivanova I, Carrieri C, Reik W, Enright AJ, O'Carroll D. 2018. Defective germline reprogramming rewires the spermatogonial transcriptome. Nat. Struct.

Peer) reviewing PDF | (2021:06:62032:1:0:NEW 25 Sep 2021) 
598

599

600

601

602

603

604

605

606

607

608

609

610

611

612

613

614

615

616

617

618

619

620

621

622

623

624

625

626

627

628

629

630

631

632

633

634

635

Mol. Biol. 25:394-404.

Weaver S, Shank SD, Spielman SJ, Li M, Muse SV, Kosakovsky Pond SL. 2018. Datamonkey 2.0: A Modern Web Application for Characterizing Selective and Other Evolutionary Processes. Mol. Biol. Evol. 35:773-777.

Wilczynska A, Minshall N, Armisen J, Miska EA, Standart N. 2009. Two Piwi proteins, Xiwi and Xili, are expressed in the Xenopus female germline. RNA 15:337-345.

Wu P-H, Fu Y, Cecchini K, Özata DM, Arif A, Yu T, Colpan C, Gainetdinov I, Weng Z, Zamore PD. 2020. The evolutionarily conserved piRNA-producing locus pi6 is required for male mouse fertility. Nature Genetics [Internet] 52:728-739. Available from: http://dx.doi.org/10.1038/s41588-020-0657-7

Wynant N, Santos D, Vanden Broeck J. 2017. The evolution of animal Argonautes: evidence for the absence of antiviral AGO Argonautes in vertebrates. Scientific Reports [Internet] 7. Available from: $h$ ttp://dx.doi.org/10.1038/s41598-017-08043-5

Yang Z. 1998. Likelihood ratio tests for detecting positive selection and application to primate Iysozyme evolution. Molecular Biology and Evolution [Internet] 15:568-573. Available from: http://dx.doi.org/10.1093/oxfordjournals.molbev.a025957

Yates AD, Achuthan P, Akanni W, Allen J, Allen J, Alvarez-Jarreta J, Amode MR, Armean IM, Azov AG, Bennett R, et al. 2020. Ensembl 2020. Nucleic Acids Res. 48:D682-D688.

Yi M, Chen F, Luo M, Cheng Y, Zhao H, Cheng H, Zhou R. 2014. Rapid evolution of piRNA pathway in the teleost fish: implication for an adaptation to transposon diversity. Genome Biol. Evol. 6:1393-1407.

Zoch A, Auchynnikava T, Berrens RV, Kabayama Y, Schöpp T, Heep M, Vasiliauskaité L, Pérez-Rico YA, Cook AG, Shkumatava A, et al. 2020. SPOCD1 is an essential executor of piRNA-directed de novo DNA methylation. Nature 584:635-639.

\section{Figure legends}

Figure 1: Phylogenetic reconstruction of the vertebrate Piwi family. A phylogenetic tree constructed from a reduced dataset to summarize relationships among Piwi paralogs. The IQTree2 model chosen was $L G+F+I+G 4$ ( $L G$ model using empirical base Frequencies, a proportion of Invariable sites, and a discrete Gamma model with 4 rate categories). A tree constructed from all species used is presented in Fig. S1. Piwi paralogs are color coded on the tree and tip labels are color coded for major gnathostome groups. The displayed tree was the maximum likelihood tree constructed with IQ-Tree2. Numbers by nodes correspond to support values from the ultrafast bootstrap routine in IQ-Tree2.

Figure 2: Synteny comparisons of vertebrate Piwil1 and Piwil4. Organization of genes up and downstream of (A) Piwil1 and (B) Piwil4. Distances are not drawn to scale. White boxes represent genes that are not homologous to any other genes in the synteny block. Boxes on top of the black line reflect genes in forward orientation relative to Piwi genes and boxes below the 
636 line are in the opposite orientation. An " $X$ " indicates pseudogenization and empty space

637 between Fut4 and Amotl1 in 2B indicates an absent Piwil4.

638 Figure 3: Evolutionary properties of Piwil3. (A) Synteny around the Piwil3 gene. White boxes

639 represent genes that are not homologous to any other genes in the synteny block, and empty

640 space between Sgsm1 and Aym1 in the mouse locus indicates a missing Piwil3. (B)

641 Evolutionary rate measured as $d_{N} / d_{S}$ mapped to branches of a mammalian Piwil1 and Piwil3

642 phylogenetic tree calculated from a free-ratio model in Codeml. (C) Codon sites under selection

643 estimated by Bayes Empirical Bayes (BEB) from Codeml model M2a. M2a sites were chosen

644 over M8 because $\mathrm{M} 2 \mathrm{a}$ is more conservative and sites under selection in M8 are also identified

645 in M2a. (D) One-ratio $d_{N} / d_{S}$ estimates among Piwis in major lineages calculated using model M0

646 in Codeml. (E) Pairwise $d_{N} / d_{S}$ distances among Piwis and major lineages.

647 Figure 4: Piwi expression characteristics. (A) Expression of Piwi genes from both somatic

648 and gonadal tissue from representatives of vertebrate lineages. Expression values were

649 normalized by taking the $\log _{10}$ of transcripts per million (TPM) +1. (B) Bar plot of Piwi expression

650 in just testes.

651 Figure 5: An evolutionary hypothesis describing the history of gene gain and loss among

652 vertebrates. The common ancestor of vertebrates had two Piwis (an ancestral Piwil1 and

653 Piwil2). Piwil2 has not undergone any gain or loss during vertebrate evolution. Most likely, Piwil4

654 was derived from a tandem duplication of Piwil1 in the common ancestor of gnathostomes, but

655 was independently lost in ray-finned fishes, birds, and monotreme mammals. Piwil3 was derived

656 from a tandem duplication of Piwil1 in the common ancestor of therian mammals.

\section{Supplemental Information}

658 Figure S1: Sample relationships and Piwi presence/absence. The phylogenetic tree was 659 generated using TimeTree (Kumar et al. 2017) from a list of species used in the study.

660 Branches are color coded by major gnathostome lineages. The presence of a Piwi paralog is 661 indicated by a red circle and absent paralog is reflected by a white circle. An $x 2$ or $x 3$ by a Piwi

662 indicates 2 or 3 copies of a paralog. Although Asterias rubens was used in the study, it is absent 663 from the tree as As. rubens relationships are lacking from TimeTree, but the Piwi

664 presence/absence mirrors Acanthaster planci and As. rubens is sister to Ac. planci (See Table 665 S1).

666 Figure S2: Full phylogenetic tree including all sequences used in the study. The IQ-Tree 667 model chosen was JTT+F+R6 (JTT model using empirical base frequencies and a FreeRate 668 model with 6 rate categories). Piwi paralogs are color coded on the tree and tip labels are color 669 coded to by major gnathostome groups. The displayed tree was constructed with IQ-Tree2.

670 Numbers next to nodes reflect nodal support derived from the ultrafast bootstrap routine/ $\mathrm{SH}$ -

671 aLRT/and posterior probability from the Bayesian phylogeny that mirrored the displayed tree. 
672 Figure S3: Full phylogenetic tree from a Gblocks filtered alignment. A phylogenetic tree 673 reconstructed from the most alignable residues see Fig S2 for additional description. The IQ-

674 Tree model chosen was also JTT+F+R6.

675 Figure S4: Piwil1 retrogene in spiny toad. (A) Location and intron/exon boundaries of Piwil1

676 paralogs in the spiny toad. (B) Piwi expression among paralogs measured from available

677 samples.

678 Figure S5: Synteny around Piwil2. Organization of genes up and downstream of Piwil2.

679 Distances are not drawn to scale. White boxes represent genes that are not homologous to any 680 other genes in the synteny block. Boxes on top of the black line reflect genes in forward 681 orientation relative to Piwi genes and boxes below the line are in the opposite orientation.

682 Figure S6: Piwi LOGO. A sequence LOGO constructed from an amino acid alignment of all 683 Piwi paralogs found in human, dog, chicken, spotted gar, and elephant shark. PAZ and PIWI 684 domains are labeled. Highly gapped regions in the 5' section were removed prior to making the 685 LOGO.

686 Figure S7: Sites under positive selection in Piwil3. All sites tested for selection in codeml 687 under model M2a presented in Figure 3C. Sites labeled by an asterisk were found under

688 selection with BEB > 0.9. PAZ and PIWI domains are highlighted.

689 Figure S8: Results of aBSREL test for episodic selection. Piwil3 branches that had 690 evidence of episodic diversifying selection are presented thicker. Significance was assessed 691 using the LRT at a threshold of $p \leq 0.05$, after correcting for multiple testing.

692 Table S1: Piwi sequence information. The list of species, common names, major lineage, and 693 transcript accession numbers for sequences used in this study.

694 Table S2: RNASeq library information. Accession numbers of RNASeq libraries used in this 695 study.

696 Table S3: Result of PAML's site test among vertebrate groups with adequate samples 697 and paralogs. Likelihood ratio tests suggest models allowing selection in codon evolution were 698 improved over models restricting selection are bolded. Codon positions where BEB > 0.9 are 699 listed, bolded positions in M8 were also found under selection in M2a.

700

Data S1. Raw Piwi DNA sequences

701 Data S2. Amino acid alignment and resulting trees used for Figure 1.

702 Data S3. Amino acid alignment and resulting trees used for Figure S2.

703 Data S4. Gblock reduced amino acid alignment and resulting trees used for Figure S3.

Data S5. Alignment used in free-ratio model (Figure 3B) and ABSREL tests (Fig S8).

Peer] reviewing PDF | (2021:06:62032:1:0:NEW 25 Sep 2021) 
705 Data S6. Alignments used for site-tests and pairwise $d_{N} / d_{S}$ distances.

706 Data S7. Raw TPM expression values for each species and tissue. 


\section{Figure 1}

Phylogenetic reconstruction of the vertebrate Piwi family.

A phylogenetic tree constructed from a reduced dataset to summarize relationships among Piwi paralogs. The IQ-Tree2 model chosen was $L G+F+I+G 4$ ( $L G$ model using empirical base Frequencies, a proportion of Invariable sites, and a discrete Gamma model with 4 rate categories). A tree constructed from all species used is presented in Fig. S1. Piwi paralogs are color coded on the tree and tip labels are color coded for major gnathostome groups. The displayed tree was the maximum likelihood tree constructed with IQ-Tree2. Numbers by nodes correspond to support values from the ultrafast bootstrap routine in IQ-Tree2. 


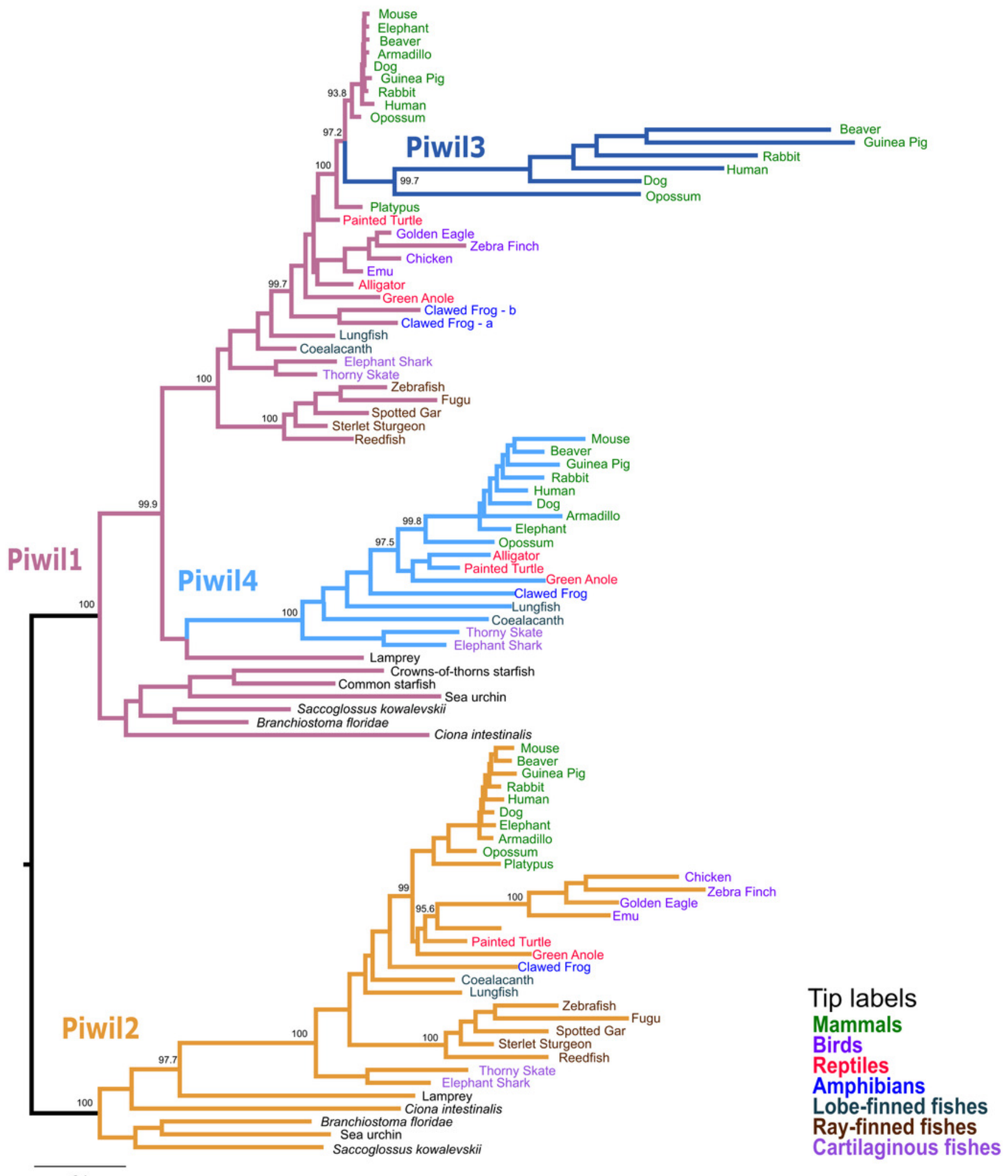


Figure 2

Synteny comparisons of vertebrate Piwil1 and Piwil4

Organization of genes up and downstream of (A) Piwil1 and (B) Piwil4. Distances are not drawn to scale. White boxes represent genes that are not homologous to any other genes in the synteny block. Boxes on top of the black line reflect genes in forward orientation relative to Piwi genes and boxes below the line are in the opposite orientation. An " $\mathrm{X}$ " indicates pseudogenization and empty space between Fut4 and Amotl1 in 2B indicates an absent

Piwil4.

A

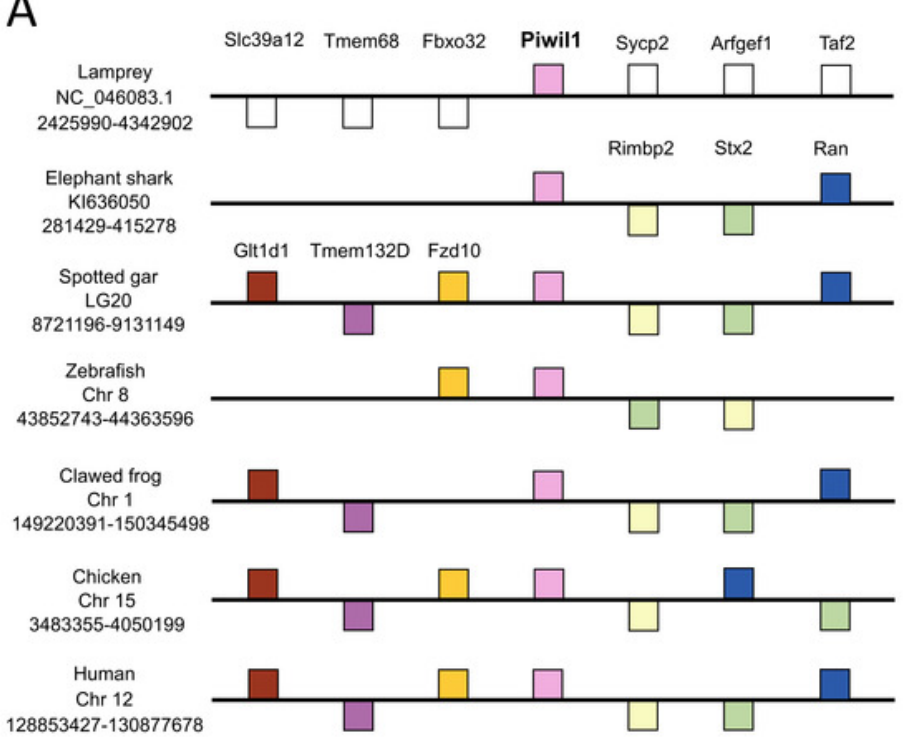

B

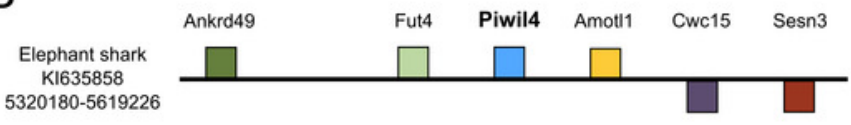

Spotted gar

LG3

12029331-12128822

Clawed frog

Chr 2

161240209-162069883

Tuatara

543691-1373847

Crocodile

MDVP01000028 84578431-185087086

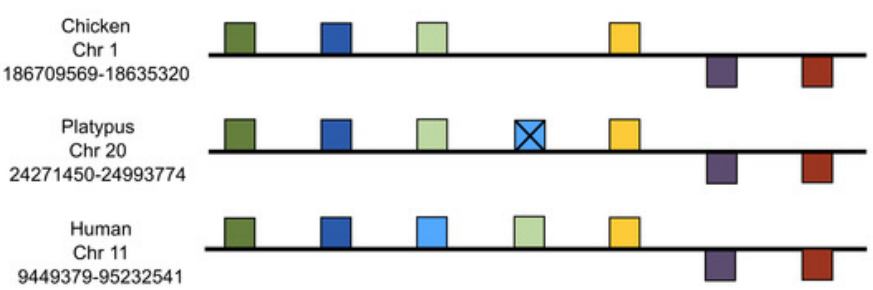




\section{Figure 3}

\section{Evolutionary properties of Piwil3}

(A) Synteny around the Piwil3 gene. White boxes represent genes that are not homologous to any other genes in the synteny block, and empty space between Sgsm1 and Aym1 in the mouse locus indicates a missing Piwil3. (B) Evolutionary rate measured as $d_{N} / d_{\mathrm{s}}$ mapped to branches of a mammalian Piwil1 and Piwil3 phylogenetic tree calculated from a free-ratio model in Codeml. (C) Codon sites under selection estimated by Bayes Empirical Bayes (BEB) from Codeml model M2a. M2a sites were chosen over M8 because M2a is more conservative and sites under selection in M8 are also identified in M2a. (D) One-ratio $d_{N} / d_{\mathrm{S}}$ estimates among Piwis in major lineages calculated using model M0 in Codeml. (E) Pairwise $d_{N} / d_{\mathrm{S}}$ distances among Piwis and major lineages. 
A

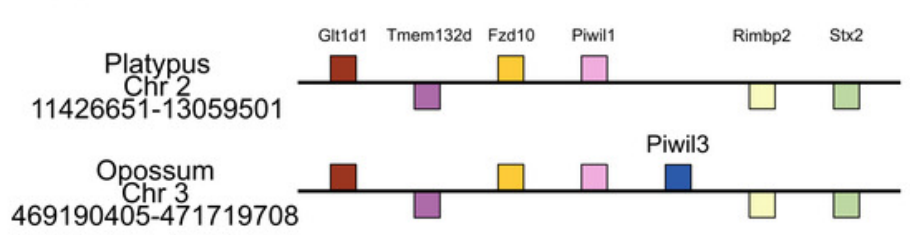

Elephant 301813-19113451
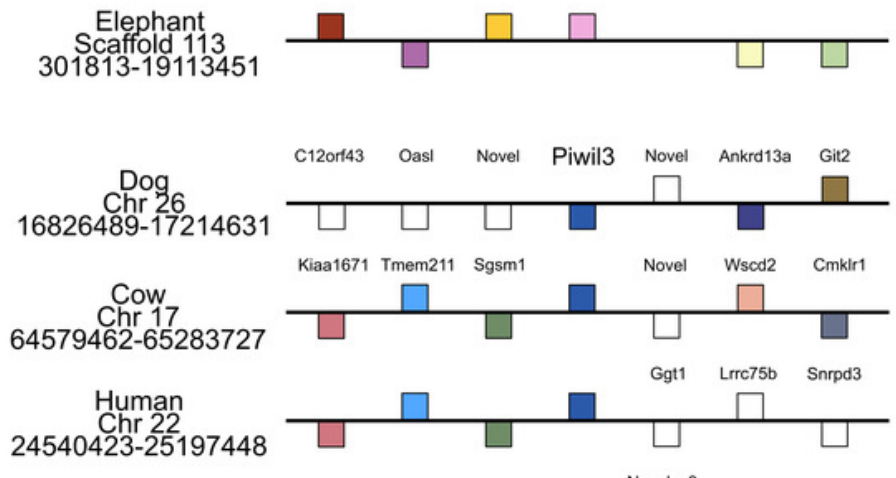

Rabbit GLO18766
74217-604548

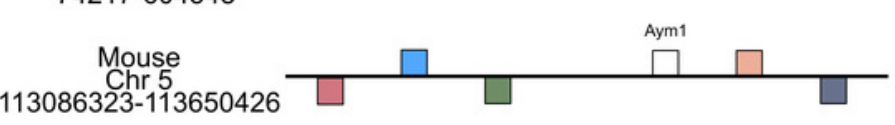

\section{D}

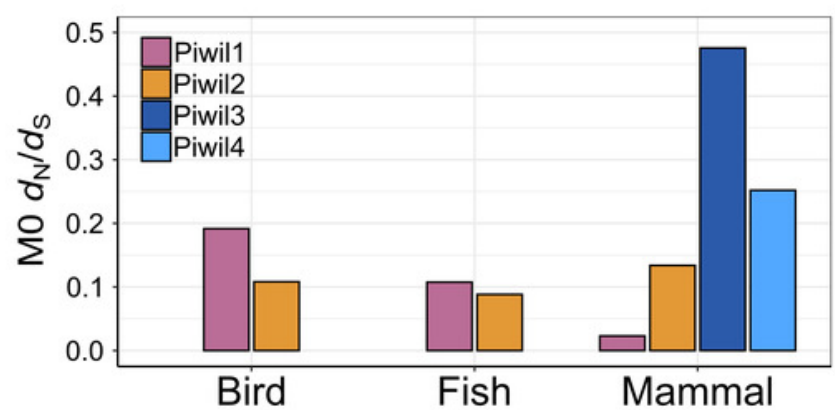

B
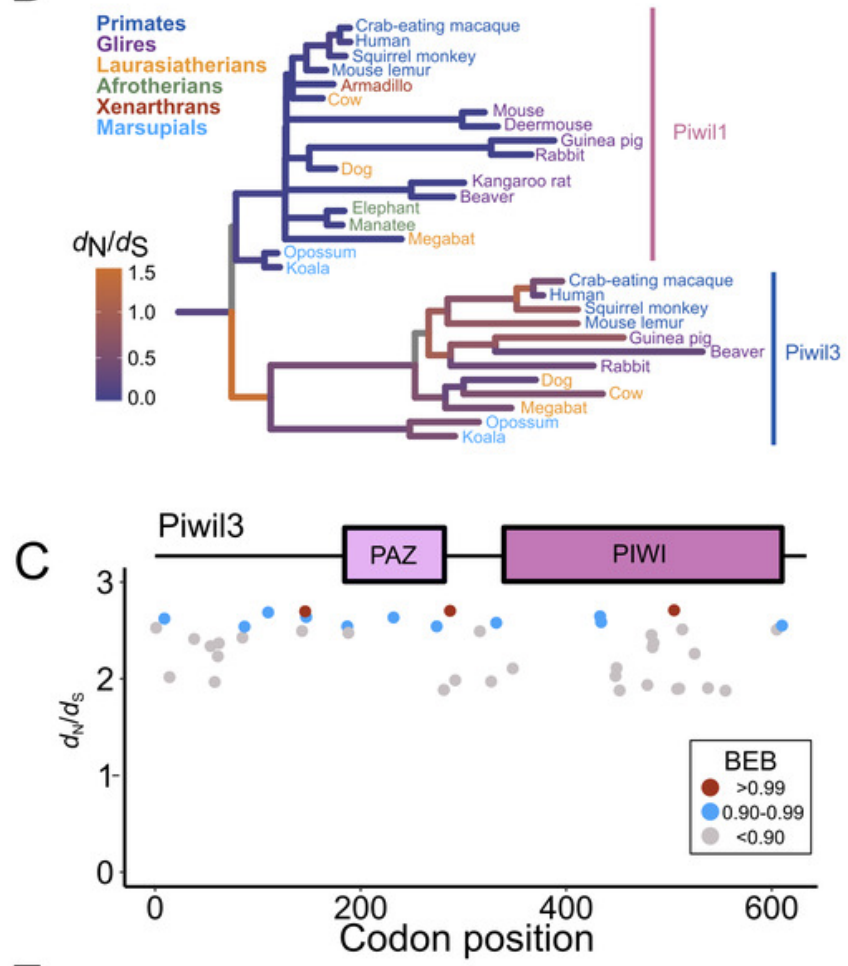

E

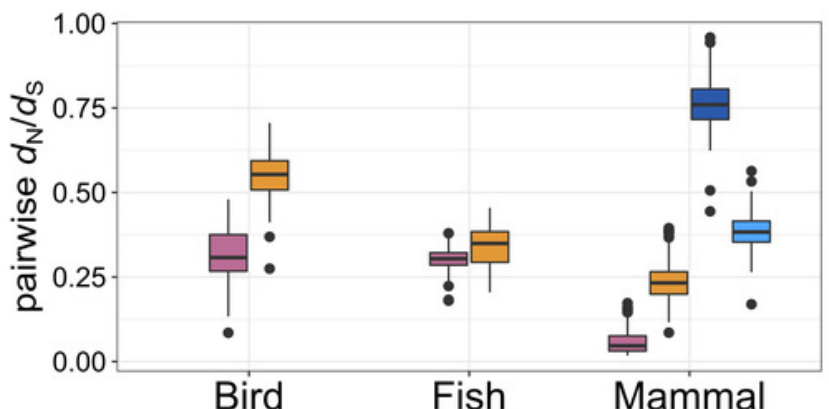


Figure 4

Piwi expression characteristics.

(A) Expression of Piwi genes from both somatic and gonadal tissue from representatives of vertebrate lineages. Expression values were normalized by taking the $\log _{10}$ of transcripts per million (TPM) +1. (B) Bar plot of Piwi expression in just testes.

A

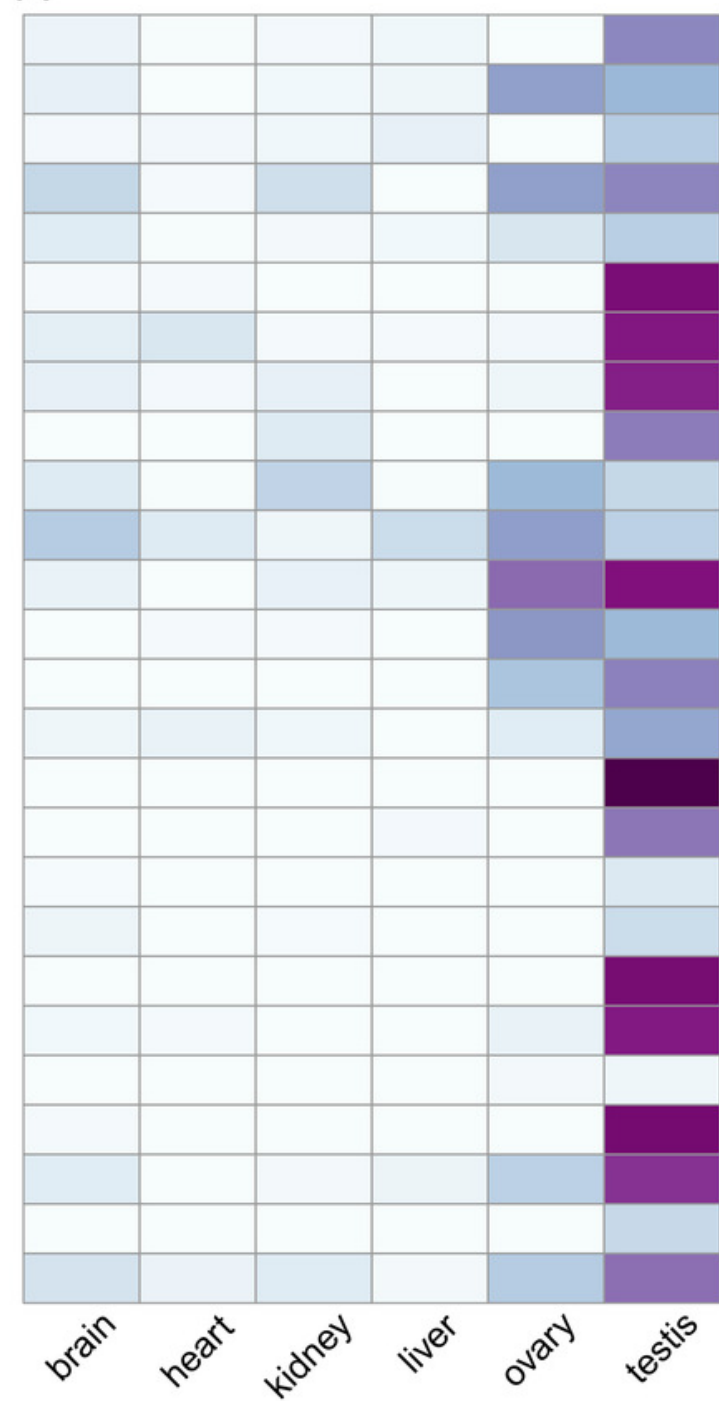

B

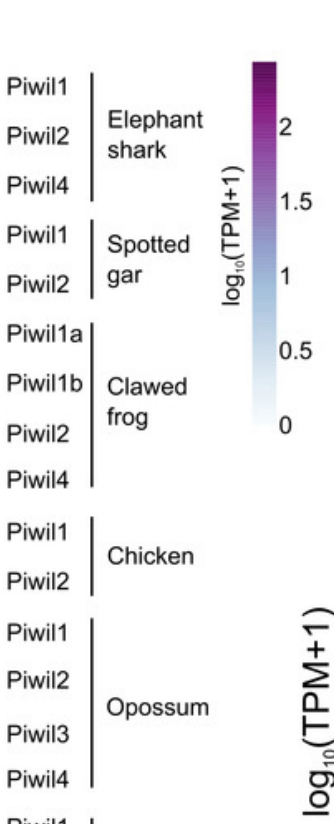

Piwil1

Piwil2

Piwil3

Cow

Piwil4

Piwil1

Piwil2 Mouse

Piwil4

Piwil1

Piwil2

Piwil3

Human

Piwil4
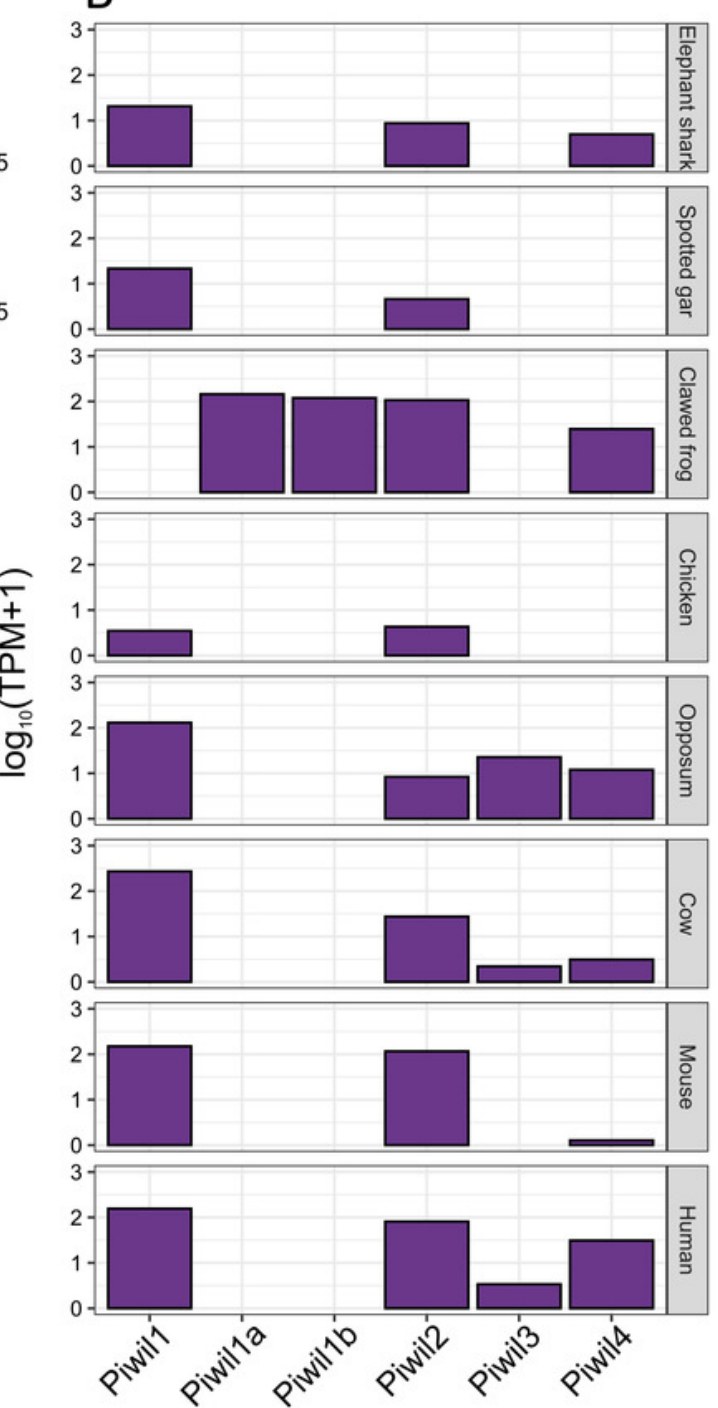


\section{Figure 5}

An evolutionary hypothesis describing the history of gene gain and loss among vertebrates.

The common ancestor of vertebrates had two Piwis (an ancestral Piwil1 and Piwil2). Piwil2 has not undergone any gain or loss during vertebrate evolution. Most likely, Piwil4 was derived from a tandem duplication of Piwil1 in the common ancestor of gnathostomes, but was independently lost in ray-finned fishes, birds, and monotreme mammals. Piwil3 was derived from a tandem duplication of Piwil1 in the common ancestor of therian mammals. 


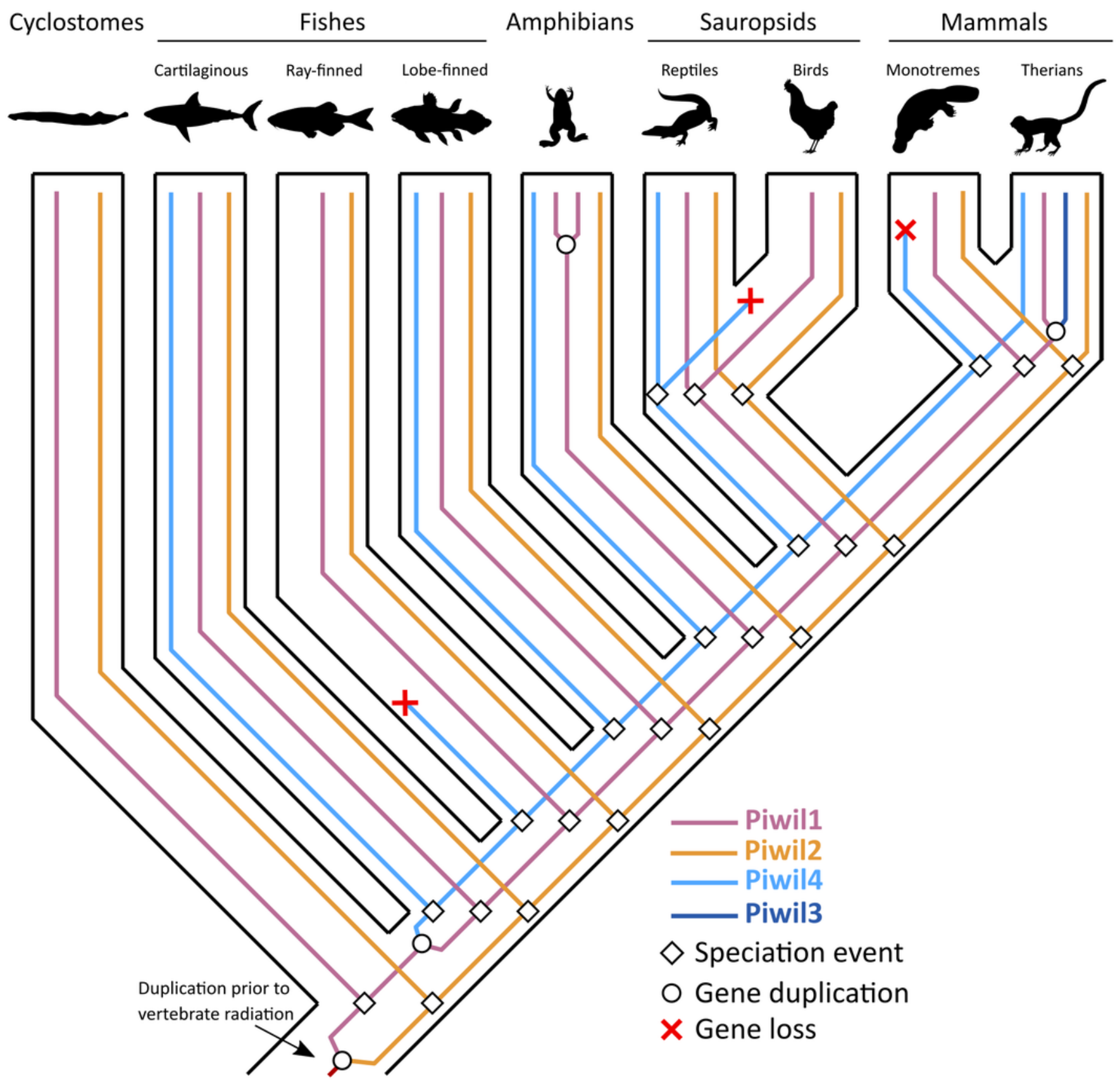

